\title{
Proteasome inhibitors reduce thrombospondin-1 release in human dysferlin-deficient myotubes
}

\author{
Esther Fernández-Simón ${ }^{1}$, Cinta Lleixà', Xavier Suarez-Calvet ${ }^{1,2}$, Jordi Diaz-Manera ${ }^{1,2,3}$, Isabel Illa $a^{1,2,3}$, \\ Eduard Gallardo ${ }^{1,2^{*}+}$ and Noemí de Luna ${ }^{1,2^{*}}$ (i)
}

\begin{abstract}
Background: Dysferlinopathies are a group of muscle disorders causing muscle weakness and absence or low levels of dysferlin, a type-II transmembrane protein and the causative gene of these dystrophies. Dysferlin is implicated in vesicle fusion, trafficking, and membrane repair. Muscle biopsy of patients with dysferlinopathy is characterized by the presence of inflammatory infiltrates. Studies in the muscle of both human and mouse models of dysferlinopathy suggest dysferlin deficient muscle plays a role in this inflammation by releasing thrombospondin1. It has also been reported that vitamin D3 treatment enhances dysferlin expression. The ubiquitin-proteasome system recognizes and removes proteins that fail to fold or assemble properly and previous studies suggest that its inhibition could have a therapeutic effect in muscle dystrophies. Here we assessed whether inhibition of the ubiquitin proteasome system prevented degradation of dysferlin in immortalized myoblasts from a patients with two missense mutations in exon 44.
\end{abstract}

Methods: To assess proteasome inhibition we treated dysferlin deficient myotubes with EB1089, a vitamin D3 analog, oprozomib and ixazomib. Western blot was performed to analyze the effect of these treatments on the recovery of dysferlin and myogenin expression. TSP-1 was quantified using the enzyme-linked immunosorbent assay to analyze the effect of these drugs on its release. A membrane repair assay was designed to assess the ability of treated myotubes to recover after membrane injury and fusion index was also measured with the different treatments. Data were analyzed using a one-way ANOVA test followed by Tukey post hoc test and analysis of variance. A $p \leq 0.05$ was considered statistically significant.

Results: Treatment with proteasome inhibitors and EB1089 resulted in a trend towards an increase in dysferlin and myogenin expression. Furthermore, EB1089 and proteasome inhibitors reduced the release of TSP-1 in myotubes. However, no effect was observed on the repair of muscle membrane after injury.

Conclusions: Our findings indicate that the ubiquitin-proteasome system might not be the main mechanism of mutant dysferlin degradation. However, its inhibition could help to improve muscle inflammation by reducing TSP-1 release.

Keywords: Dysferlin, Proteasome, Vitamin D3, Myogenin, Sarcolemma, Thrombospondin-1

\footnotetext{
* Correspondence: egallardo@santpau.cat; nluna@santpau.cat

${ }^{\dagger}$ Eduard Gallardo and Noemí de Luna contributed equally to this work.

'Neuromuscular Diseases group. Institut de Recerca Hospital de Sant Pau,

Biomedical Research Institute Sant Pau (IIB Sant Pau), Universitat Autónoma

de Barcelona, c/Sant Antoni Ma Claret 167, 08025 Barcelona, Spain

Full list of author information is available at the end of the article
}

C C The Author(s). 2020 Open Access This article is licensed under a Creative Commons Attribution 4.0 International License, which permits use, sharing, adaptation, distribution and reproduction in any medium or format, as long as you give appropriate credit to the original author(s) and the source, provide a link to the Creative Commons licence, and indicate if changes were made. The images or other third party material in this article are included in the article's Creative Commons licence, unless indicated otherwise in a credit line to the material. If material is not included in the article's Creative Commons licence and your intended use is not permitted by statutory regulation or exceeds the permitted use, you will need to obtain permission directly from the copyright holder. To view a copy of this licence, visit http://creativecommons.org/licenses/by/4.0/ The Creative Commons Public Domain Dedication waiver (http://creativecommons.org/publicdomain/zero/1.0/) applies to the data made available in this article, unless otherwise stated in a credit line to the data. 


\section{Background}

Mutations in the dysferlin gene (DYSF) that lead to absence or marked reduction of the protein are the cause of dysferlinopathy [1]. The disease is an autosomal recessive muscle disorder and has several phenotypes, including limb girdle 2B [2], Miyoshi myopathy [3] and distal anterior compartment myopathy [4]. Progression of the disease is characterized by muscle weakness and atrophy, and muscle biopsy shows mainly necrosis of muscle fibers and inflammatory infiltrates. Although several genetic and pharmacologic treatments have been tested $[5,6]$, no curative treatment is yet available for dysferlinopathy [7].

DYSF contains 55 exons and 14 isoforms. Fifty-three percent of patients have nonsense mutations, $43 \%$ have missense mutations, and $4 \%$ have small in-frame insertions or deletions [1]. While nonsense mutations produce a complete absence of the protein, a residual expression of dysferlin is detected in primary myotubes from patients carrying missense mutations $[8,9]$.

Dysferlin protein is highly expressed in skeletal muscle, cardiac muscle and blood monocytes [10]. It is a type-II transmembrane protein that contains seven $\mathrm{C} 2$ domains $\left(\mathrm{Ca}^{2+}\right.$-binding $)$ involved in vesicle fusion, trafficking, membrane repair and regulation of calcium homeostasis $[11,12]$. However, it is also associated with other processes, including intracellular signaling and myoblast differentiation [13]. Studies using primary cultures of human skeletal muscle show that dysferlin and myogenin may share a pathway involved in differentiation of skeletal muscle "in vitro" as dysferlin-deficient (dysf mut/mut) myotubes have reduced expression of myogenin and are poorly differentiated [14]. It has been reported that thrombospondin-1 (TSP-1) expression is increased in dysf mut/mut myotubes and may play a role in the inflammation observed in muscle biopsies of patients with dysferlin myopathy [15]. In fact, it has been shown that serum TSP1 levels correlate with macrophage inflammation and muscle damage in dysferlin-deficient BlaJ mice [16].

The ubiquitin-proteasome system (UPS) is the major proteolytic pathway as more than $80 \%$ of cellular proteins are degraded therein [17]. The UPS is an ATPdependent system that removes unfolded or misfolded proteins [18]. It interacts only with proteins labelled with a polyubiquitin chain [18]. Proteins bind proteasome through the $\alpha$-rings of the $20 \mathrm{~S}$ subunit and then pass through the $\beta$-rings, where they are degraded by the proteasome particles: chymotrypsin-like (CT-L), caspaselike (C-L), and trypsin-like (T-L) proteolytic sites [19].

Previous studies have shown that proteasome inhibitors could have a therapeutic implication in muscle dystrophies. It has been reported that the proteasome inhibitor MG-132 rescued the expression of the dystrophin-glycoprotein complex (DGC) in mouse models of Duchenne muscular dystrophy and in skeletal muscle cultures from patients with
Duchenne and Becker muscular dystrophy [20-22]. Bortezomib, another proteasome inhibitor, promoted the expression and membrane localization of dystrophin and dystrophin-associated proteins in $m d x$ mice, but the drug had only a modest effect in myoblasts from patients with DMD [22]. Since patients carrying missense mutations in DYSF have residual expression of dysferlin, prevention of its degradation by the UPS could be used as a therapeutic approach.

Apart from avoiding protein degradation, other studies have focused on increasing dysferlin expression by activating gene expression. In peripheral blood monocytes (PBM) and myotubes of dysferlinopathy carriers bearing one mutation in DYSF, dysferlin expression is increased after vitamin D3 treatment [8]. Vitamin D3 binds to the vitamin D3 response element (VDRE) present in the dysferlin promoter and enhances dysferlin expression. EB1089 is an analogue of vitamin D3 that shows higher efficacy and less hypercalcemic activity [23], but its effect on dysferlin expression has not been studied.

As there are no effective treatments for dysferlinopathies, we assessed whether inhibition of the ubiquitin proteasome system could prevent degradation of dysferlin in immortalized myoblasts from a patient carrying two missense mutations. We used oprozomib and ixazomib, $2 \mathrm{~s}$ generation proteasome inhibitors that have reduced toxicity and off-targets, increasing effectiveness. We also studied the role of EB1089 in the expression of dysferlin, alone or in combination with proteasome inhibitor. We evaluated the effect of these treatments by studying the expression of myogenin, the ability of myotubes to repair the sarcolemma after injury and the release of TSP-1.

\section{Material and methods Samples}

In all experiments we used immortalized myoblasts from a dysferlinopathy patient with homozygous missense mutations (c.4882G > A/p.G1628R) in exon 44 of DYSF and from a healthy control. This patient corresponds to number 16 in our previous work and was described showing some degree of dysferlin expression in sarcoplasm [24]. These cells present a residual expression of dysferlin of about 1-2\% compared to WT myotubes. These cells were kindly provided by Dr. Mouly [25]. Myoblasts were expanded using skeletal muscle medium (SMM; Promocell, Heidelberg, Germany) until confluence was achieved. Media was then changed to differentiation medium (75\% Dulbecco's Modified Eagle's Medium and 25\% M199, supplemented with 2\% FBS (Lonza, Basel, Switzerland), $10 \mu \mathrm{g} / \mathrm{ml}$ insulin (Sigma-Aldrich, St Louis, MO, USA), $2 \mathrm{mmol} / \mathrm{l}$ glutamine (Lonza) and penicillinstreptomycin (Lonza)) for 7 to 9 days. Differentiated myotubes were treated with vitamin D3 or EB1089 (Sigma 
Aldrich) at $100 \mathrm{nM}$, ixazomib (Selleckchem, Munich, Germany) (25 nM, $50 \mathrm{nM}$ and $100 \mathrm{nM})$ and oprozomib (ONX 0912)(Selleckchem) (10 nM, $50 \mathrm{nM}$ and $100 \mathrm{nM})$. Ixazomib and oprozomib were combined with EB1089 at $8 \mathrm{~h}$ and $24 \mathrm{~h}$, respectively.

\section{Western blot}

Cell pellets corresponding to each condition were lysed in RIPA buffer (Sigma-Aldrich) containing a protease and phosphatase inhibitor cocktail (Roche, Basel, Switzerland). Lysates were centrifuged at $4{ }^{\circ} \mathrm{C}$ at $13000 \mathrm{x}$ $g$ for $20 \mathrm{~min}$ and supernatants were stored at $-80^{\circ} \mathrm{C}$. Protein concentrations were determined using Pierce ${ }^{\text {tx }}$ BCA Protein Assay (Thermo Fisher Scientific, Waltham, MA, USA). Thirty micrograms of protein were resolved in a $10 \%$ sodium dodecyl sulfate (SDS) polyacrylamide gel and transferred to nitrocellulose membranes. Unspecific binding sites were blocked by incubation for $1 \mathrm{~h}$ in casein diluted 1:1 in tris-buffered saline (TBS). Blots were incubated overnight with the primary mouse monoclonal antibodies anti-dysferlin (NCL-Hamlet, Novocastra, Newcastle, UK) and anti-myogenin (5FD clone) (Santa Cruz Biotechnology, Dallas, TX). To normalize the results, mouse anti-desmin (Novocastra) was added simultaneously with the primary antibody. The secondary antibody for anti-dysferlin was a goat anti-mouse labeled with IR-Dye 800 (Li-Cor, Lincoln, Nebraska, USA). When the anti-myogenin antibody was used as a primary antibody, biotin-labeled horse antimouse (Jackson ImmunoResearch, Ely, UK) was used as a secondary antibody. Secondary antibodies were incubated for $1 \mathrm{~h}$ at room temperature. Membranes incubated with biotinylated secondary antibody were washed and incubated for $1 \mathrm{~h}$ with IRDye-680-labeled streptavidin (Li-Cor). After extensive washing, the immunoreactive bands were visualized using the Odyssey Infrared ImagingSystem (Li-Cor). The amount of protein was quantified using Image Studio Lite software (Li-Cor). Desmin expression was used as a loading control. As a reference value we used dysferlin or myogenin expression of the healthy control. Protein expression in the remaining conditions was quantified over the expression in WT myotubes and expressed as fold-change.

\section{Assessment of proteasome-like activity}

Cells were seeded in a 96- well black plate (Sarstedt, Nümbrecht, Germany) at 5000 cells/well in 3 replicates until confluence. The media was then changed to differentiation medium. Cells were treated with the corresponding drug for the indicated time. The CT-L, C-T and $\mathrm{T}-\mathrm{L}$ activity was assayed by chemiluminiscence using the Proteasome-Glo ${ }^{\text {ma }} 3$-substrate System cell based assay (Promega, Madison, WI, USA) and the plate was read using Victor 3v Multilabel Plate Reader (Perkin Elmer, Waltham, MA, USA).

\section{Thrombospondin-1 Enzyme-Linked Immunosorbent Assay (ELISA)}

Immortalized myoblasts from the dysferlinopathy patient were seeded at 5000 cells $/ \mathrm{cm}^{2}$ and expanded until confluence. The media was then changed to differentiation medium to form myotubes. After treatment with proteasome inhibitors together with or without EB1089, we removed the media and added $1 \mathrm{ml}$ of basal DMEM (Lonza) to the culture for $24 \mathrm{~h}$. Cell culture supernatants were concentrated using Amicon Ultra Centrifugal Filters $100 \mathrm{~kb}$ (Merck Millipore, Darmstadt, Germany). TSP-1 present in the culture media was detected using the human TSP-1 Immunoassay (Quanti-Kine ELISA, R\&D Systems, Minneapolis, MN), following the manufacturer's instructions. The detection limit of the assay was $0.355 \mathrm{ng} / \mathrm{mL}$.

\section{Membrane repair assay}

Immortalized myoblasts from the dysferlinopathy patient and the healthy control were grown until confluence in chambered wells and then differentiated to myotubes. The assay was performed as recently described in human muscle primary cultures [26]. SDS is an anionic surfactant that causes the leakage of intracellular components due to its ability to affect membranes [27]. Membrane injury was induced by detergent treatment as follows: after the cell cultures were washed with Hank's Balanced Salt Solution (HBSS) (Lonza), the injury solution (HBSS with $0.12 \mathrm{mM}$ or $0.25 \mathrm{mM}$ SDS (Sigma-Aldrich)) was applied for $2 \mathrm{~min}$. Following exposure to injury solution, cells were washed in HBSS and then incubated in recovery solution consisting of proliferation media for $90 \mathrm{~s}$ and $10 \mathrm{~min}$. The injury and recovery steps were performed at $37^{\circ} \mathrm{C}$. Cells with injured permeable membranes were identified by exposure to propidium iodide (PI) (Sigma-Aldrich) $(20 \mu \mathrm{g} / \mathrm{mL}$ in HBSS). If cell membrane is not repaired, PI enters the cell and penetrates the nucleus wherein it intercalates with DNA and emits fluorescence. After $2 \mathrm{~min}$ of exposure to the dye, cells were washed with HBSS and fixed with $4 \%$ paraformaldehyde in PBS. Nuclei were stained with Hoescht 33,342 (Invitrogen, Thermo-Fisher Scientific). In each experimental condition, we counted the total number of nuclei and the PI positive nuclei using Fiji software [28].

\section{Fusion index}

Once membrane repair assay was performed, myotubes were permeabilized with ethanol for $5 \mathrm{~min}$ at room temperature. Samples were then blocked with the UltraCruz Blocking Reagent (Santa Cruz Biotechnology, Dallas, TX) for $1 \mathrm{~h}$ at RT. Myotubes were stained using a mouse anti-myosin heavy chain (MyHC) antibody (MF- 
20)(Bio-Rad, Hercules, CA, USA) for $1 \mathrm{~h}$ at RT and then the goat anti-mouse secondary antibody Alexa Fluor 594 (ThermoFisher, Wal- tham, MA) was used as a secondary antibody for $1 \mathrm{~h}$ at RT. Following the incubation with the secondary antibody, nuclei were stained with Hoescht 3342 (Invitrogen, Thermo-Fisher Scientific). Images were acquired and analysed using Fiji software.

The fusion index was determined as the percentage of nuclei included in MF-20-expressing myotubes (containing at least 3 nuclei) divided by the total number of nuclei.

\section{Statistical analysis}

Multiple comparisons were analyzed using a one-way ANOVA test followed by Tukey post hoc test. When statistical comparisons were performed between two groups, the nonparametric Mann-Whitney test was used. GraphPad Prism 5.0 software was used (LaJolla, CA, USA) for graphic representation.

\section{Results}

\section{EB1089 and vitamin D3 treatment}

Vitamin D3 and EB1089 were added to healthy control myotubes for $24 \mathrm{~h}$ at $100 \mathrm{nM}$ in order to assess their effect on dysferlin expression. Both Vitamin D3 and EB1089 increased dysferlin expression. However, only treatment with EB1089 showed statistically significant differences compared to non-treated myotubes (Fig. 1). Therefore, from this point on, we decided to use EB1089 in combination with proteasome inhibitors to increase dysferlin expression.

\section{Proteasome inhibition}

We analyzed the time points at which ixazomib and oprozomib produced the highest increase in dysferlin expression in dysf mut/mut myotubes. The effect of ixazomib was highest at $8 \mathrm{~h}$ and the effect of oprozomib was highest at $24 \mathrm{~h}$ (data not shown). We also analyzed whether the addition of EB1089 to the proteasome inhibitors had an effect on dysferlin expression at the same time points.

Treatment of dysf mut/mut myotubes with $10 \mathrm{nM}$ oprozomib for $24 \mathrm{~h}$ showed high selectivity towards the $\mathrm{CT}-\mathrm{L}$ active site of the proteasome. However, at higher doses (50 $\mathrm{nM}$ and $100 \mathrm{nM}$ ) oprozomib also inhibited C$\mathrm{L}$ and $\mathrm{T}-\mathrm{L}$ activity significantly (Fig. 2a-c).

Ixazomib treatment inhibited all 3 proteasome subunits (Fig. 2d-f).

When EB1089 was added alone to the culture, no proteasome inhibition in any subunit was found. Moreover, when it was combined with ixazomib and oprozomib, no effect was observed in the inhibition of each subunit of the proteasome.

\section{Dysferlin and myogenin expression}

We analyzed the expression of dysferlin and myogenin in dysf mut/mut myotubes treated with EB1089, oprozomib and ixazomib.

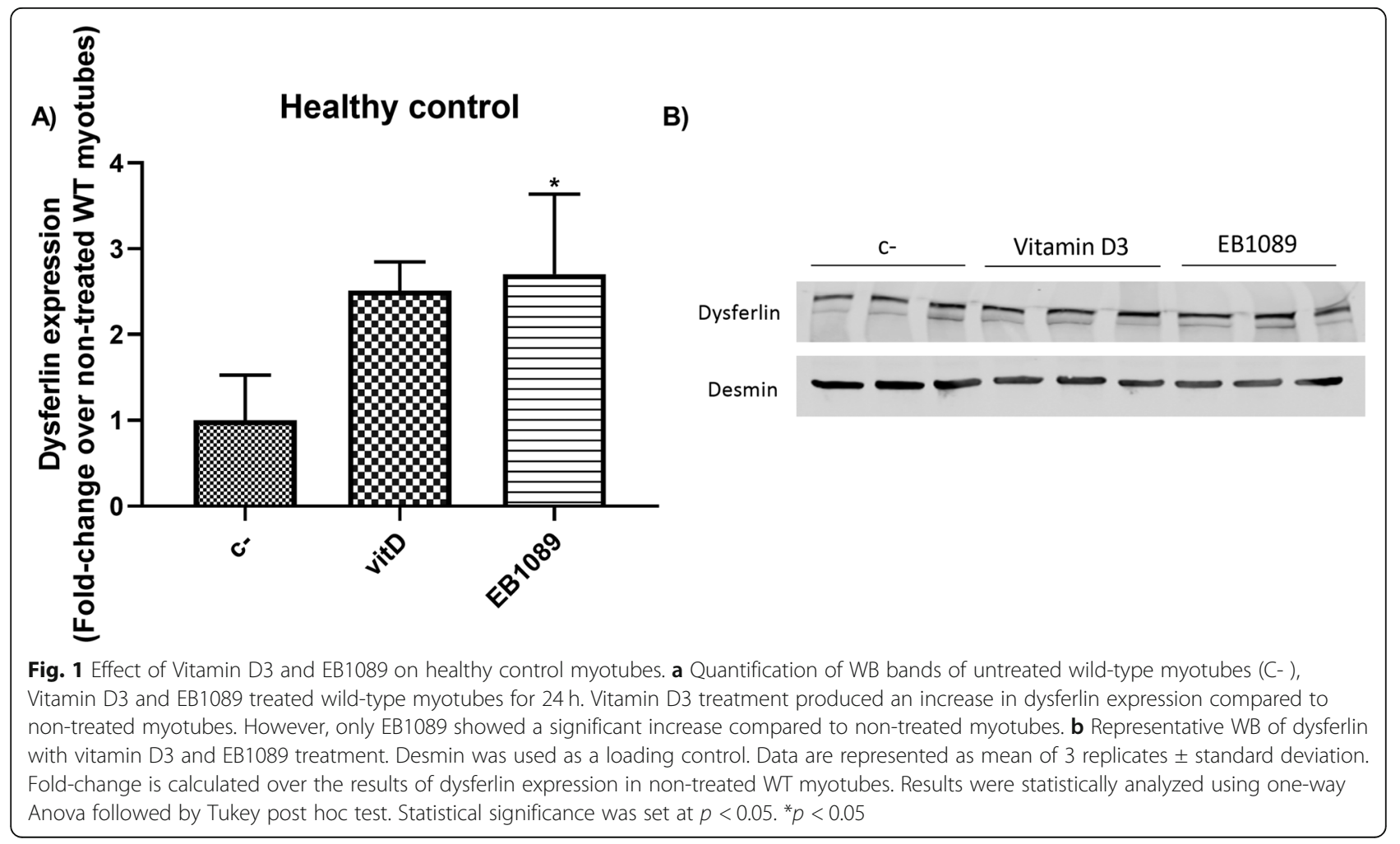




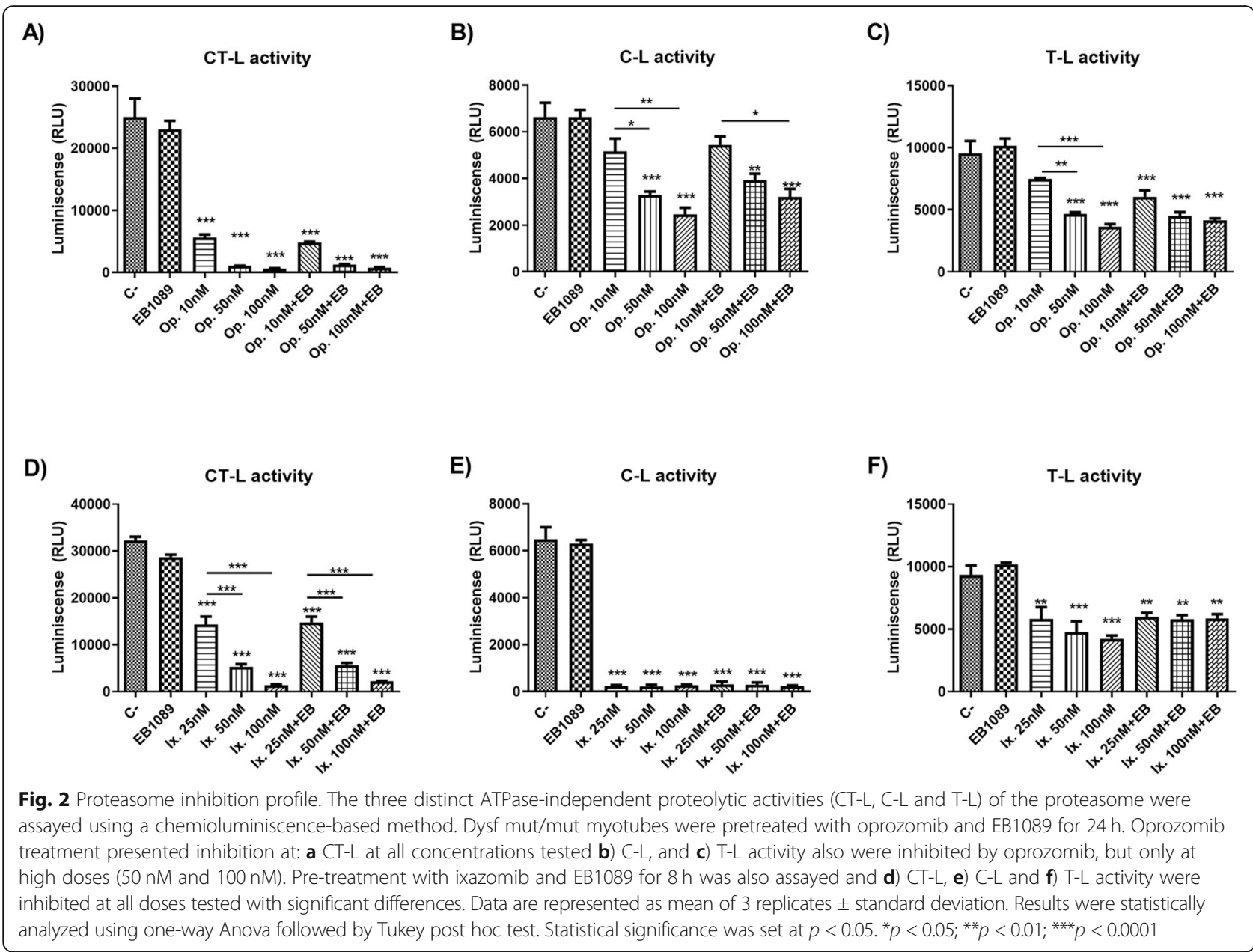

Treatment with oprozomib at $24 \mathrm{~h}$ showed a trend towards an increase in myogenin expression (Fig. 3a-b). When EB1089 was added together with oprozomib, myogenin presented a trend towards decreased expression compared to oprozomib treatment alone. Dysferlin presented a trend towards increased expression with $10 \mathrm{nM}$ of oprozomib with and without EB1089. No statistical significances were observed in any experimental conditions.

Treatment with ixazomib (Fig. 3c-d) showed no significant increase in the expression of dysferlin. Myogenin levels showed a trend towards increased expression after ixazomib treatment, being $100 \mathrm{nM}$ the concentration at which the myogenin expression was the highest. The addition of EB1089 together with ixazomib presented a trend towards decreased expression of myogenin compared to ixazomib treatment alone, but the results did not reach statistical significance.

\section{Expression of TSP-1}

We evaluated the secretion of TSP-1 by dysf mut/mut myotubes treated with EB1089, oprozomib and ixazomib. Treatment with EB1089 significantly reduced TSP-
1 release at $8 \mathrm{~h}$. TSP- 1 levels also decreased significantly in dysf mut/mut cells treated with oprozomib and EB1089 separately (Fig. 4a). TSP-1 levels decreased even more significantly when cells were treated with the combination of EB1089 and oprozomib.

Ixazomib treatment significantly decreased TSP-1 only when combined with EB1089 (Fig. 4b). However, although EB1089 and ixazomib alone did not produce significant changes in TSP-1 expression, expression was lower than in untreated myotubes.

Raw western-blot data are available in Supplementary Figure 1.

\section{Sarcolemma repair assay}

Considering that dysferlin is involved in membrane repair after injury, we analyzed the ability of dysf mut/mut myotubes, treated with proteasome inhibitors and EB1089, to repair the sarcolemma after injury. We found that wild-type myotubes repaired the membrane at both low and high concentration of SDS $(0.12 \mathrm{mM}$ SDS and at $0.25 \mathrm{mM}$ SDS), showed by PI exclusion, $10 \mathrm{~min}$ after injury (Fig. 5a). However, dysf mut/mut myotubes did 


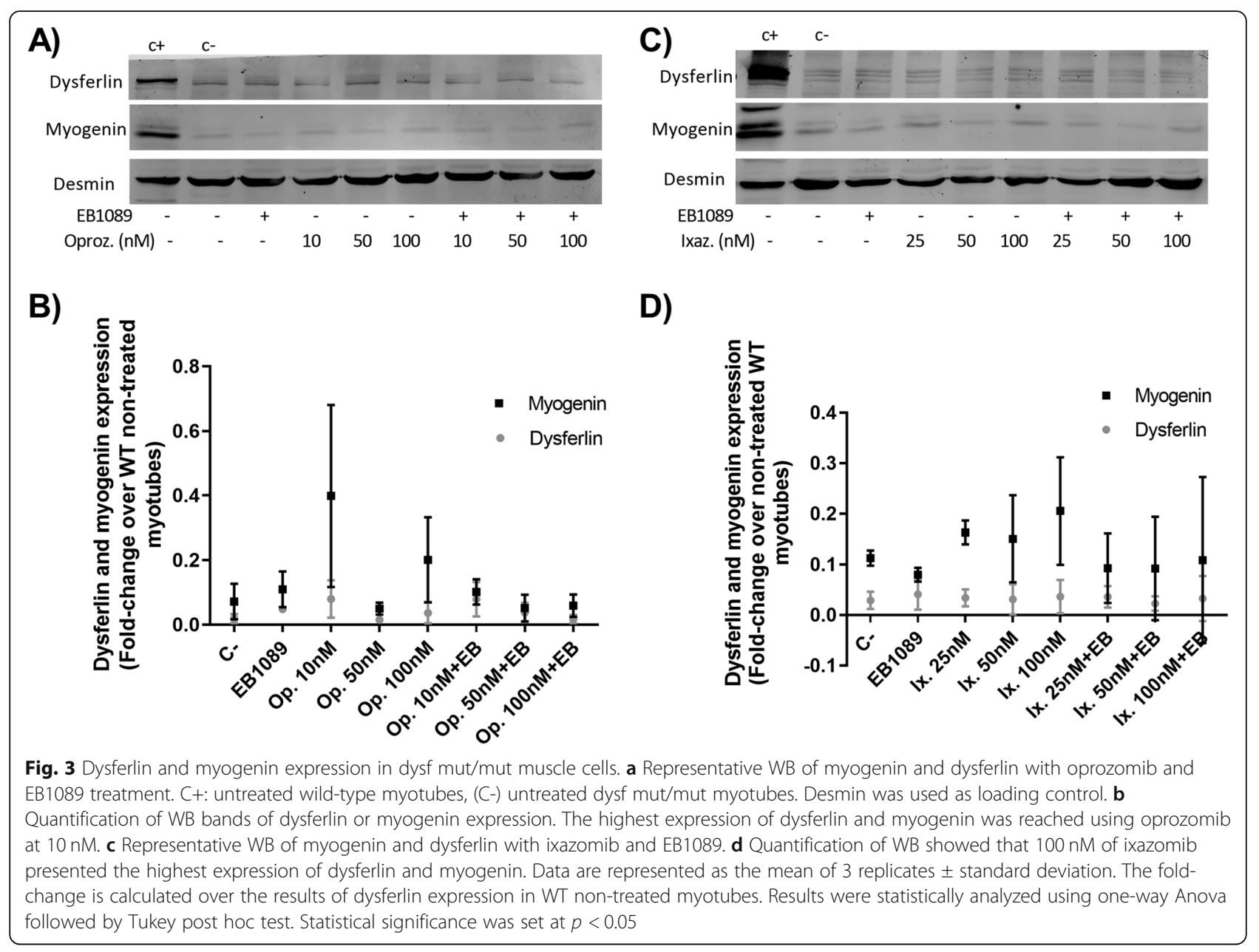

not present any sarcolemma repair in any condition tested (Fig. 5a-c).

\section{Fusion index}

Fusion index results showed that dysf mut/mut myotubes presented a statistically significant lower fusion index compared to wild-type myotubes (Fig. 5e). Ixazomib, oporozomib and EB1089 did not show any effect on the fusion index of dysf mut/mut myotubes (Fig. 5f-g).

\section{Discussion}

The aim of this study was to test if blockade of proteasome could restore dysferlin expression and consequently rescue the muscle functions that are impaired when dysferlin is missing. It has been reported that dysferlin deficient myoblasts display reduced levels of myogenin and consequently fusion index is reduced [14]. Muscles biopsies of dysferlinopathy patients are characterized by the presence of abundant inflammatory infiltrates [29], TSP-1 seems to have a prominent role in muscle inflammation in this pathology [15]. Finally, dysferlin is involved in sarcolemma repair $[26,30]$.
Treatment with oprozomib, ixazomib and EB1089 in myotubes from a dysferlinopathy patient promoted a trend towards increased expression of dysferlin and myogenin, but this low increase did not translate into higher sarcolemmal repair neither higher fusion index than that in untreated myotubes. However, TSP-1 release from myotubes decreased after treatment.

Western blot analysis showed a trend towards increased expression of dysferlin and myogenin when oprozomib and EB1089 were added to the culture. Ixazomib produced a trend towards increased expression of myogenin but not dysferlin. These results are in agreement with a previous work by Fujita E et al. They found that mutant-dysferlin was mainly degraded by autophagy while wild type-dysferlin was degraded by the ubiquitin-proteasome system, although they used a proteasome inhibitor different from those used in this study [31]. The small increase in dysferlin expression that we observed after treating dysf mut/mut myotubes with our different ubiquitin-proteasome inhibitors confirms that this system is not the main pathway to recycle mutated dysferlin. 
A)

\section{Dysf mut/mut}

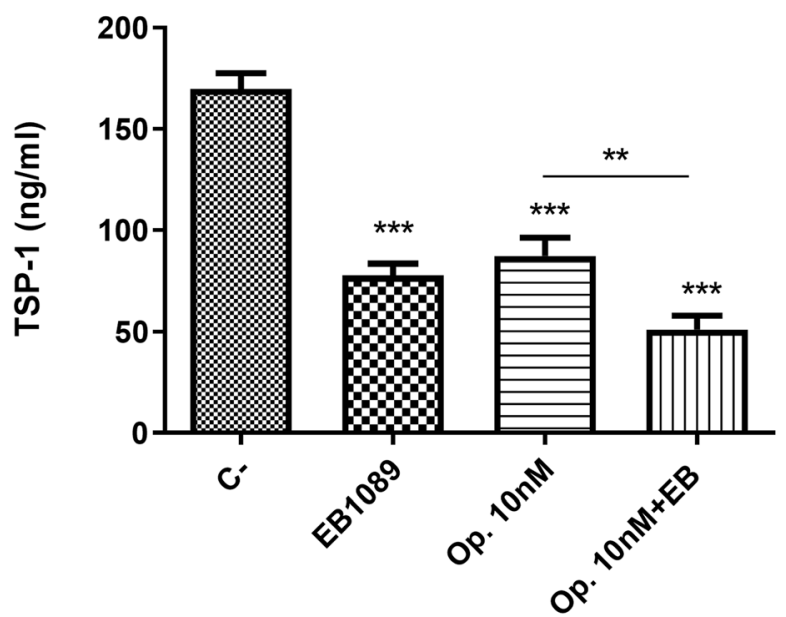

B)

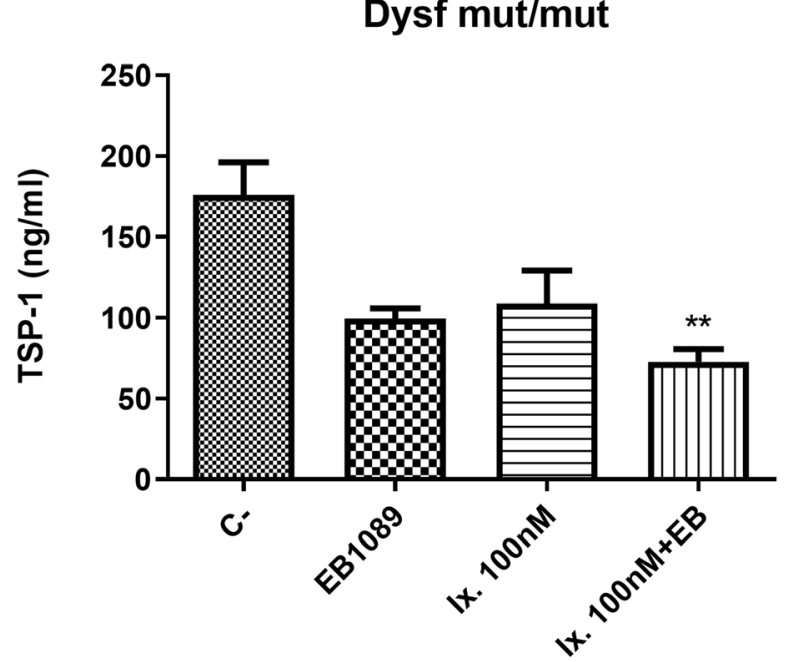

Fig. 4 TSP-1 expression by dysf mut/mut myotubes treated with oprozomib, ixazomib and EB1089. a TSP-1 expression with treatment of oprozomib at $10 \mathrm{nM}$ and EB1089 $100 \mathrm{nM}$ was significantly decreased compared to C- (non-treated dysf mut/mut myotubes), and the combination of both treatments was also significantly decreased compared to oprozomib treatment alone. $\mathbf{b}$ There were no significant differences in TSP-1 levels between ixazomib and EB1089 compared to non-treated myotubes. However, the combination of both treatments showed a significant reduction compared to non-treated myotubes. Data are represented as mean of 5 replicates \pm standard deviation. Results were statistically analyzed using one-way Anova followed by Tukey post hoc test. Statistical significance was set at $p<0.05$. ${ }^{* *} p<0.01$; ${ }^{* * *} p<0.0001$

The other treatment that we studied, the analogue of Vitamin D3, EB1089, produced a trend towards increased expression of dysferlin. In other studies, authors found that Vitamin D3 treatment had no effect on dysferlin expression in myotubes from dysferlinopathy patients but increased dysferlin expression in muscle cultures from carriers of one mutation in DYSF [8]. Although EB1089 is 50-200 times more potent than vitamin D3 [23], when added to cultured myotubes from a dysferlinopathy patient it only produces a trend towards an increase in dysferlin expression. Vitamin D3 acts through the Vitamin D receptor and p38 MAPK and participates in differentiation events in skeletal muscle cells [32]. Whether this effect is dependent on dysferlin expression remains to be elucidated. Moreover, EB1089 also presented a trend towards decreased expression of myogenin when was combined with proteasome inhibitors compared to the results of treatment with a proteasome inhibitor alone. Although results were not statistically significant, this negative effect could be explained by a negative regulation of myogenin after EB1089 treatment. In previous studies, authors showed that Vitamin D3 downregulates the expression of myogenin both in vitro and in vivo [33-35]. These previous findings suggest that negative vitamin $\mathrm{D}$ response elements may be present in the promoter region of myogenin, however those have not been identified [36].

We also analyzed the expression of TSP-1 in cell cultures after treatment with proteasome inhibitors. TSP-1 is secreted in response to inflammation and multiple factors modulate its release. It is expressed by endothelial cells, fibroblasts, neutrophils, macrophages, $\mathrm{T}$ cells, and myotubes [37]. We observed a significant decrease in TSP-1 release when dysf mut/mut myotubes were exposed to EB1089, oprozomib and the combination of oprozomib or ixazomib and EB1089. The decrease in TSP-1 levels could be the consequence of the trend towards an increased expression of dysferlin in these conditions. However, TSP-1 levels were significantly lower when a combination of oprozomib and EB1089 was added to the culture than when oprozomib was added alone, suggesting that proteasome inhibitors and EB1089 act independently of dysferlin expression to reduce TSP1 release.

The reduced expression of TSP-1 observed after treatment with EB1089 could be explained by mechanisms independent of dysferlin expression. Amarasekera AT et al. suggested that vitamin $\mathrm{D}$ supplementation reduces TSP-1 levels in healthy individuals [38]. Moreover, it has been studied that calcitrol, a vitamin D metabolite, downregulates TSP-1 via the activation of the mitogen activated protein kinase (MAPK) [39]. Therefore, EB1089 treatment could activate the MAPK pathway leading to downregulation of TSP-1. This activation could constitute an alternative mechanism to reduce TSP-1 levels that is not linked to dysferlin expression.

The effect of proteasome inhibitors on TSP-1 could also be explained by mechanisms non-related to dysferlin expression, such as the inhibition of canonical NF- $\mathrm{KB}$ 


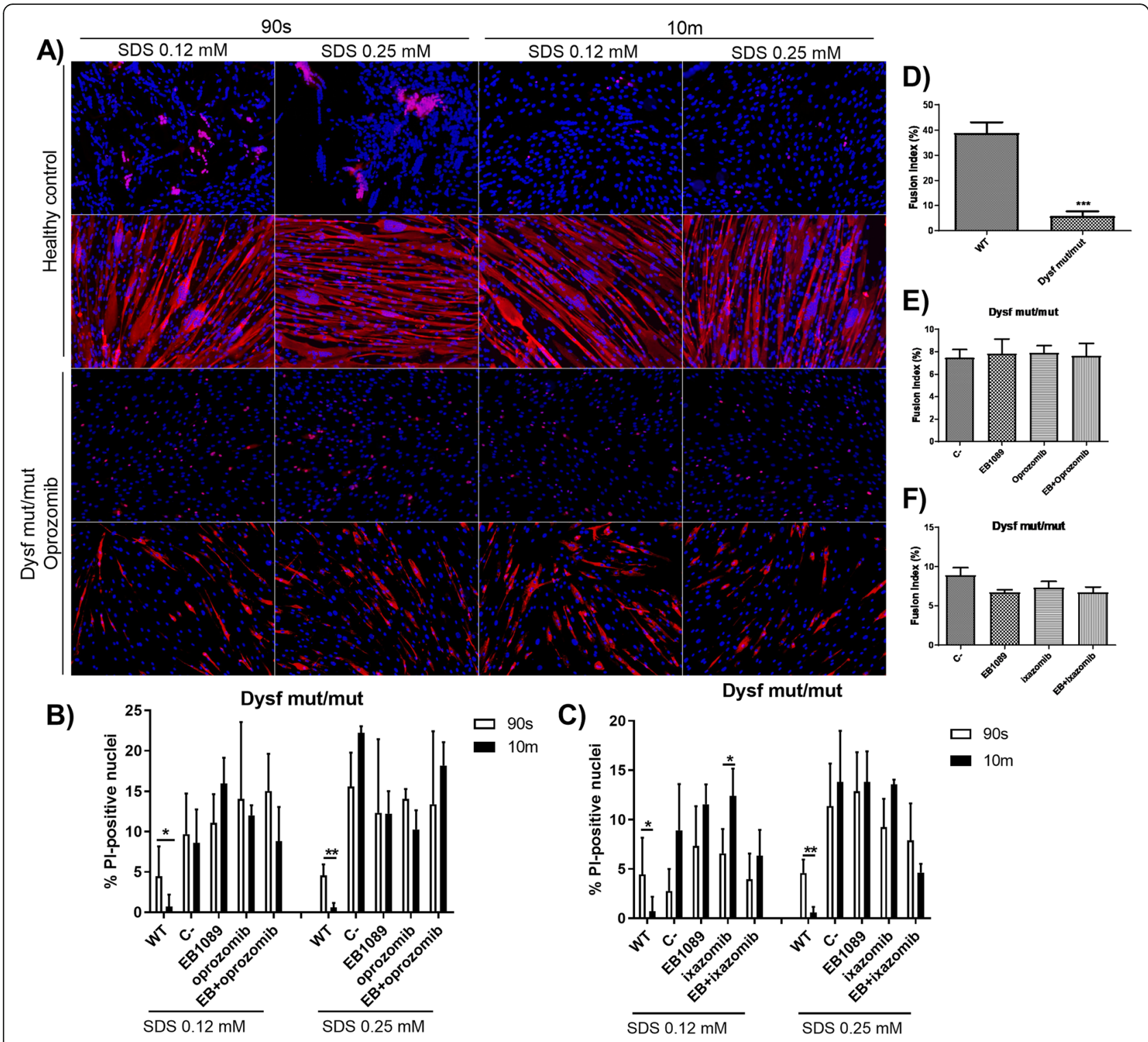

Fig. 5 Membrane repair and fusion index using oprozomib, ixazomib and EB1089 treatment. a Representative images of PI-positive nuclei and MyHC positive myotubes after SDS treatment at $90 \mathrm{~s}$ and at $10 \mathrm{~min}$. The first 2 rows correspond to healthy myotubes and the last 2 rows correspond to dysf mut/mut myotubes treated with oprozomib. b Quantification of IP-positive nuclei in myotubes from healthy controls and dysf mut/mut myotubes treated with EB1089, oprozomib, and the combination of oprozomib $10 \mathrm{nM}$ with EB1089at at $24 \mathrm{~h}$. c Quantification of IPpositive nuclei in myotubes from healthy controls and dysf mut/mut myotubes treated with EB1089, ixazomib, and the combination of ixazomib $100 \mathrm{nM}$ with EB1089at at $8 \mathrm{~h}$. Differences were not observed with any treatment. d Quantification of fusion index showed a statistically significant decrease of $\mathrm{MyHC}$ positive myotubes compared to healthy myotubes. e Fusion index in dysf mut/mut myotubes treated with EB1089, oprozomib at $10 \mathrm{nM}$ and the combination of oprozomib $10 \mathrm{nM}$ with EB1089 at $8 \mathrm{~h}$ did not show any difference compared to non-treated myotubes. $\mathbf{f}$ Fusion index in dysf mut/mut myotubes treated with EB1089, ixazomib at $100 \mathrm{nM}$ and the combination of ixazomib $100 \mathrm{nM}$ with EB1089 at $24 \mathrm{~h}$ did not show any difference compared to non-treated myotubes. Data are represented as mean of 3 replicates \pm standard deviation. Results were statistically analyzed using Mann-Whitney test to compare two groups and one-way Anova followed by Tukey post hoc test for multiple comparisons. Statistical significance was set at $p<0.05$. ${ }^{*} p<0.05 ;{ }^{* *} p<0.01 ;{ }^{* *} p<0.0001$

signaling. NF- $\mathrm{B}$ f forms a dimer with IкB, an inhibitory protein, which keeps the complex inactive in the cytoplasm. When signals activate this pathway, ІкB is phosphorylated and degraded by the proteasome, releasing the NF- $\mathrm{kB}$ to the nucleus where it regulates the transcription of genes involved in the inflammatory response. Proteasome inhibitors seem to decrease the nuclear levels of NF- $\mathrm{kB}$ by avoiding the degradation of I $\kappa B$, hence decreasing NF- $\kappa B$ activity [40]. In effect, in dysferlinopathy patients, damage-associated molecular patterns (DAMPs) are released from damaged muscle fibers, triggering inflammatory responses via activation 


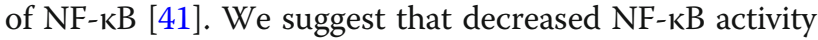
by proteasome inhibitors could be another mechanism involved in TSP-1 reduction and thereby reduce the inflammatory response.

We did not observe any sarcolemma repair when proteasome inhibitors and EB1089 were added to the culture. We have previously reported that dysferlin mut/ mut cells fuse less efficiently than wild-type myoblasts. We also demonstrated that dysferlin mut/mut myotubes showed a reduced expression of myogenin in the nucleus [14]. The experiments performed in the present study confirm that fusion of myoblasts is impaired in dysferlin mut/mut. Lack of dysferlin impaires sarcolemmal repair both in myoblasts and myotubes therefore, we believe that reduced fusion of myoblasts has no effect on the membrane repair experiments. The trend towards increased expression of dysferlin after treatment was not strong enough to restore sarcolemma repair of damaged myotubes.

\section{Conclusions}

In conclusion, we did not observe a significant increase of dysferlin or myogenin in myotubes from a dysferlinopathy patient when treated with EB1089, oprozomib and ixazomib. However, we did observe a significant reduction in TSP-1 levels. Targeting TSP-1 may constitute a therapeutic approach in patients carrying mutations affecting proper folding of dysferlin since it would reduce inflammation and avoid the undesirable side-effects reported in patients with dysferlinopathy treated with prednisone.

\section{Supplementary Information}

The online version contains supplementary material available at https://doi. org/10.1186/s12891-020-03756-7.

Additional file 1: Supplementary Figure 1.' Dysferlin and myogenin expression in dysf mut/mut muscle cells. A) Representative full blot of dysferlin with vitamin D3 and EB1089 treatment. Desmin was used as a loading control. B) Representative full blot of myogenin and dysferlin with oprozomib and EB1089 treatment. C) Representative full blot of myogenin and dysferlin with ixazomib and EB1089 treatment.

\section{Abbreviations \\ DYSF: Dysferlin; C2: Ca ${ }^{2+}$-binding; Dysf mut/mut: Dysferlin deficient; TSP- 1: Thrombospondin 1; UPS: Ubiquitin-proteasome system; C-L: Caspase-like; T-L: Trypsin-like; CT-L: Chemotrypsin-like; DGC: Dystrophin-glycoprotein complex; PBM: Peripheral blood monocytes; VDRE: Vitamin D3 response element; PI: Propidium iodide; DAMP: Damage-associated molecular pattern}

\section{Acknowledgements}

Not applicable.

\section{Authors' contributions}

EFS performed the experiments, analysed of the results, and prepared the manuscript. CLI performed the experiments. XSC analysed the results. JDM reviewed the manuscript and obtained funding. II reviewed the manuscript and obtained funding. EG performed the experimental design, analysed of the results, prepared the manuscript and obtained funding for the study. $\mathrm{NdL}$ performed the experimental design, analyzed of the results, prepared the manuscript and obtained funding for the study. The author(s) read and approved the final manuscript.

\section{Funding}

This project has been funded by projects from the Fundación Isabel Gemio to II, EG and JDM and by Fundación Ramón Areces (CIVP18A3903) to NdL. XSC was supported by a "Sara Borrell" post- doctoral fellowship project "CD18/00195", funded by Instituto de Salud Carlos III and co-funded by European Union (ERDF/ESF, "A way to make Europe"/"Investing in your future"). CERCA Programme / Generalitat de Catalunya.

\section{Availability of data and materials}

The datasets used and/or analyzed during the current study are available from the corresponding author on reasonable request.

\section{Ethics approval and consent to participate}

The study was approved by the Ethics Committee at Hospital de la Santa Creu i Sant Pau (HSCSP) in Barcelona. All patients and controls gave informed consent to use their muscle biopsy for research purposes.

\section{Consent for publication}

Not applicable.

\section{Competing interests}

The authors declare that they have no competing interests.

\section{Author details}

${ }^{1}$ Neuromuscular Diseases group. Institut de Recerca Hospital de Sant Pau, Biomedical Research Institute Sant Pau (IIB Sant Pau), Universitat Autónoma de Barcelona, c/Sant Antoni Ma Claret 167, 08025 Barcelona, Spain. ${ }^{2}$ Centro de Investigación Biomédica en Red de Enfermedades Raras (CIBERER), Valencia, Spain. ${ }^{3}$ Department of Neurology, Neuromuscular Diseases Unit, Hospital de la Santa Creu i Sant Pau, Universitat Autònoma de Barcelona, Barcelona, Spain.

Received: 9 December 2019 Accepted: 30 October 2020

Published online: 27 November 2020

\section{References}

1. Bashir R, Strachan T, Keers S, Stephenson A, Mahjneh I, Marconi G, et al. A gene for autosomal recessive limb-girdle muscular dystrophy maps to chromosome 2p. Hum Mol Genet. 1994;3(3):455-7.

2. Bashir R, Keers S, Strachan T, Passos-Bueno R, Zatz M, Weissenbach J, et al. Genetic and physical mapping at the limb-girdle muscular dystrophy locus (LGMD2B) on chromosome 2p. Genomics. 1996;33(1):46-52.

3. Bejaoui K, Hirabayashi K, Hentati F, Haines JL, Hamida C. Ben, Belal S, et al. linkage of Miyoshi myopathy (distal autosomal recessive muscular dystrophy) locus to chromosome 2p12-14. Neurology. 1995;45(4):768-72.

4. Illa I, Serrano-Munuera C, Gallardo E, Lasa A, Rojas-Garca R, Palmer J, et al. Distal anterior compartment myopathy: a dysferlin mutation causing a new muscular dystrophy phenotype. Ann Neurol. 2001;49(1):130-4.

5. Malcher J, Heidt L, Goyenvalle A, Escobar H, Marg A, Beley C, et al. Exon Skipping in a Dysf-Missense Mutant Mouse Model. Mol Ther Nucleic Acids. 2018;13:198-207.

6. Lee JJA, Maruyama R, Duddy W, Sakurai H, Yokota T. Identification of novel antisense-mediated exon skipping targets in DYSF for therapeutic treatment of Dysferlinopathy. Mol Ther Nucleic Acids. 2018;13:596-604.

7. Brown RH. Dysferlinopathies. Handb Clin Neurol. 2011;101:111-8.

8. De Luna N, Díaz-Manera J, Paradas C, Iturriaga C, Rojas-García R, Araque J, et al. 1a,25(OH)(2)-vitamin D3 increases dysferlin expression in vitro and in a human clinical trial. Mol Ther. 2012;20(10):1988-97.

9. Woolger N, Bournazos A, Sophocleous RA, Evesson FJ, Lek A, Driemer B, et al. Proteolysis reveals dysferlin conformation in situ limited proteolysis as a tool to probe the tertiary conformation of dysferlin and structural consequences of patient missense variant L344P. J Biol Chem. 2017;292(45): 18577-91.

10. Anderson LV, Davison K, Moss JA, Young C, Cullen MJ, Walsh J, et al. Dysferlin is a plasma membrane protein and is expressed early in human development. Hum Mol Genet. 1999;8(5):855-61.

11. Kerr JP, Ward CW, Bloch RJ. Dysferlin at transverse tubules regulates Ca (2+) homeostasis in skeletal muscle. Front Physiol. 2014;5:89. 
12. Abdullah N, Padmanarayana M, Marty NJ, Johnson CP. Quantitation of the calcium and membrane binding properties of the C2 domains of Dysferlin Biophysj. 2014;106:382-9.

13. Lukyanenko V, Muriel JM, Bloch RJ. Coupling of excitation to Ca2+ release is modulated by dysferlin. J Physiol. 2017;595(15):5191-207.

14. De Luna N, Gallardo E, Soriano M, Dominguez-Perles R, De La Torre C, RojasGarcía R, et al. Absence of dysferlin alters myogenin expression and delays human muscle differentiation "in vitro.". J Biol Chem. 2006;281(25):17092-8.

15. De Luna N, Gallardo E, Sonnet C, Chazaud B, Dominguez-Perles R, SuarezCalvet $X$, et al. Role of Thrombospondin 1 in macrophage inflammation in Dysferlin myopathy. J Neuropathol Exp Neurol. 2010;69(6):643-53.

16. Urao N, Mirza RE, Corbiere TF, Hollander Z, Borchers CH, Koh TJ. Thrombospondin-1 and disease progression in dysferlinopathy. Hum Mol Genet. 2017;26(24):4951-60.

17. Konstantinova IM, Tsimokha AS, Mittenberg AG. Role of Proteasomes in Cellular Regulation. Int Rev Cell Mol Biol. 2008;267:59-124 Academic Press.

18. Ciechanover A. EMBO MEMBER'S REVIEW The ubiquitin-proteasome pathway: on protein death and cell life. EMBO J. 1998;17(24):7151-60.

19. Glickman MH, Ciechanover A. The ubiquitin-proteasome proteolytic pathway: Destruction for the sake of construction. Physiol Rev. 2002;82:373428 American Physiological Society.

20. Bonuccelli G, Sotgia F, Schubert W, Park DS, Frank PG, Woodman SE, et al. Proteasome inhibitor (MG-132) treatment of $\mathrm{mdx}$ mice rescues the expression and membrane localization of dystrophin and dystrophinassociated proteins. Am J Pathol. 2003;163(4):1663-75.

21. Assereto S, Stringara S, Sotgia F, Bonuccelli G, Broccolini A, Pedemonte M, et al. Pharmacological rescue of the dystrophin-glycoprotein complex in Duchenne and Becker skeletal muscle explants by proteasome inhibitor treatment. Am J Physiol Physiol. 2006;290(2):C577-82.

22. Gazzerro E, Assereto S, Bonetto A, Sotgia F, Scarfi S, Pistorio A, et al. Therapeutic potential of proteasome inhibition in Duchenne and Becker muscular dystrophies. Am J Pathol. 2010;176(4):1863-77.

23. Lu L, Qiu J, Liu S, Luo W. Vitamin D3 analogue EB1089 inhibits the proliferation of human laryngeal squamous carcinoma cells via p57. Mol Cancer Ther. 2008;7(5):1268-74

24. Gallardo E, de Luna N, Diaz-Manera J, Rojas-García R, Gonzalez-Quereda L, Flix $B$, et al. Comparison of dysferlin expression in human skeletal muscle with that in monocytes for the diagnosis of dysferlin myopathy. PLoS One. 2011;6(12):e29061.

25. Mamchaoui K, Trollet C, Bigot A, Negroni E, Chaouch S, Wolff A, et al. Immortalized pathological human myoblasts: towards a universal tool for the study of neuromuscular disorders. Skelet Muscle. 2011;1:1(1)

26. De Luna N, Suarez-Calvet X, Garicano M, Fernandez-Simon E, Rojas-García R, Diaz-Manera J, et al. Effect of MAPK inhibition on the differentiation of a Rhabdomyosarcoma cell line combined with CRISPR/Cas9 technology: an in vitro model of human muscle diseases. J Neuropathol Exp Neurol. 2018; 77(10):964-72.

27. Malik JK, Schwarz LR, Wiebel FJ. Assessment of membrane damage in continuous cultures of mammalian cells. Chem Biol Interact. 1983;45(1):29-42.

28. Schindelin J, Arganda-Carreras I, Frise E, Kaynig V, Longair M, Pietzsch T, et al. Fiji: an open-source platform for biological-image analysis. Nat Methods. 2012;9(7):676-82.

29. Selcen $D$, Stilling G, Engel AG. The earliest pathologic alterations in dysferlinopathy. Neurology. 2001;56(11):1472-81.

30. Han R, Campbell KP. Dysferlin and muscle membrane repair. Curr Opin Cell Biol. 2007;19:409-16 NIH Public Access.

31. Fujita E, Kouroku Y, Isoai A, Kumagai H, Misutani A, Matsuda C, et al. Two endoplasmic reticulum-associated degradation (ERAD) systems for the novel variant of the mutant dysferlin: ubiquitin/proteasome ERAD(I) and autophagy/lysosome ERAD (II). Hum Mol Genet. 2007;16(6):618-29.

32. Irazoqui AP, Heim NB, Boland RL, Buitrago CG. 1a,25 dihydroxi-vitamin $D_{3}$ modulates CDK4 and CDK6 expression and localization. Biochem Biophys Res Commun. 2015:459(1):137-42

33. Van Der Meijden K, Bravenboer N, Dirks NF, Heijboer AC, Den Heijer M, De Wit GMJ, et al. Effects of 1,25(OH) 2 D 3 and 25(OH) D 3 on C2C12 myoblast proliferation, differentiation, and Myotube hypertrophy. J Cell Physiol. 2016; 231:2517-28.

34. Sydney JEG, South Wales N. Westmead Hospital, Sydney, New South Wales 2145, Australia; and St Vincent's Clinical School. Aust Endocrinology. 2010; 2052:347-57.
35. Endo I, Inoue D, Mitsui T, Umaki Y, Akaike M, Yoshizawa T, et al. Deletion of Vitamin D Receptor Gene in Mice Results in Abnormal Skeletal Muscle Development with Deregulated Expression of Myoregulatory Transcription Factors. Endocrinology. 2003;144(12):5138-44.

36. Wagatsuma A, Sakuma K. Vitamin D signaling in Myogenesis: potential for treatment of sarcopenia. Biomed Res Int. 2014;2014:121254.

37. Lopez-Dee Z, Pidcock K, Gutierrez LS. Thrombospondin-1: multiple paths to inflammation. Mediat Inflamm. 2011;2011:296069.

38. Amarasekera AT, Assadi-Khansari B, Liu S, Black M, Dymmott G, Rogers NM, et al. Vitamin D supplementation lowers thrombospondin-1 levels and blood pressure in healthy adults. PLoS One. 2017;12(5):e0174435.

39. García-Quiroz J, Rivas-Suárez M, García-Becerra R, Barrera D, Martínez-Reza I, Ordaz-Rosado D, et al. Calcitriol reduces thrombospondin-1 and increases vascular endothelial growth factor in breast cancer cells: implications for tumor angiogenesis. J Steroid Biochem Mol Biol. 2014;144:215-22.

40. Verbrugge SE, Scheper RJ, Lems WF, de Gruijl TD, Jansen G. Proteasome inhibitors as experimental therapeutics of autoimmune diseases. Arthritis Res Ther. 2015;17(1):17.

41. Mariano A, Henning A, Han R. Dysferlin-deficient muscular dystrophy and innate immune activation. FEBS J. 2013:280(17):4165-76.

\section{Publisher's Note}

Springer Nature remains neutral with regard to jurisdictional claims in published maps and institutional affiliations.
Ready to submit your research? Choose BMC and benefit from:

- fast, convenient online submission

- thorough peer review by experienced researchers in your field

- rapid publication on acceptance

- support for research data, including large and complex data types

- gold Open Access which fosters wider collaboration and increased citations

- maximum visibility for your research: over $100 \mathrm{M}$ website views per year

At BMC, research is always in progress.

Learn more biomedcentral.com/submissions 